\title{
LA PERSPECTIVA GLOTOPOLITICA EN EL ESTUDIO DE LOS INSTRUMENTOS LINGÜÍSTICOS: ASPEC- TOS TEÓRICOS Y METODOLÓGICOS
}

Elvira Narvaja de Arnoux (Universidad Nacional de Buenos Aires - UBA)

\section{RESUMEN}

El artículo parte de considerar la Glotopolítica como el estudio de las intervenciones en el espacio del lenguaje, que participan en la reproducción o transformación de las sociedades. Así como esas intervenciones dependen de los procesos sociales inciden también en ellos desde determinadas posiciones que el análisis busca identificar. Los instrumentos lingüísticos, que se proponen regular las prácticas, son enfocados como gestos glotopolíticos, de allí la necesidad de atender a sus condiciones de producción y de circulación. El trabajo aborda en ese marco y con particular referencia a gramáticas y retóricas, aspectos teóricos - la relación de la Glotopolítica con la Historiografía Lingüística, las temporalidades que pueden ser convocadas en el análisis y los espacios considerados, la importancia del intertexto en la interpretación, la función social de los dispositivos normativos y la construcción de las subjetividades- y metodológicos -la construcción de series, el análisis contrastivo de textos y la reformulación.

PALABRAS CLAVE: Glotopolítica; instrumentos lingüísticos; gramáticas; retóricas; análisis contrastivo 
La Glotopolítica estudia las intervenciones en el espacio del lenguaje, entendidas estas en un sentido amplio ya que pueden ser planificadas, explícitas, voluntarias, generadas por agentes - colectivos o individuales - que podemos identificar, o producidas "espontáneamente" sin mediadores claramente identificables. Estas intervenciones se ejercen tanto sobre las lenguas, sus variedades y registros como sobre los discursos o las diversas articulaciones de lo verbal con otros sistemas semióticos. En todos los casos podemos reconocer la incidencia de los procesos sociales en el espacio del lenguaje y la impronta de las tecnologías de la palabra, asociadas con aquellos. Pensemos, por ejemplo, en el estallido de géneros o la desacralización de registros vinculados con procesos revolucionarios; o en el tuiteo, resultado de las tecnologías globales con las restricciones que este formato impone y la representación de la comunicación que lo sostiene.

Los hechos del lenguaje que interrogamos se exponen en textos como manuales de estilo, ensayos sobre la lengua, gramáticas escolares, notas periodísticas normativas- o en prácticas o, incluso, en esos condensados significativos que son las escenas, en su función tanto de ilustración de una situación general como de problematización de lo aceptado (ARNOUX, 2014a). En el análisis intentamos fundamentalmente dilucidar la dimensión política de los fenómenos abordados estudiándolos como intervenciones en el espacio público del lenguaje que tienden a establecer (reproducir o transformar) un orden social, modelando a la vez las identidades, es decir, construyendo las subjetividades necesarias en cada instancia histórica.

En todas las situaciones interrogamos las ideologías lingüísticas (ARNOUX y DEL VALLE, 2010) en las que se apoyan o con las que están asociadas las diferentes intervenciones. Entendemos las ideologías lingüísticas como sistemas de representaciones acerca de objetos lingüísticos diversos que van, por ejemplo, del acento regional al modo de lectura privilegiado en la escuela o en la red. Esas representaciones con su fuerte dimensión valorativa son puestas en relación con los procesos en curso, las posiciones sociales o los posicionamientos dentro de un campo. De allí la importancia de los datos contextuales en el análisis.

Por cierto que muchas prácticas discursivas, propias de distintos ámbitos y épocas, han generado dispositivos normativos que cristalizan en instrumentos lingüísticos destinados a regular las hablas, como los diccionarios, las gramáticas, o las retóricas. A partir de ejemplos referidos a 
estos dos últimos instrumentos voy a reflexionar en este artículo deteniéndome en algunos aspectos teóricos - la relación de la Glotopolítica con la Historiografía Lingüística, las temporalidades que pueden ser convocadas en el análisis y los espacios considerados, la importancia del intertexto en la interpretación, la función social de los dispositivos normativos y la construcción de las subjetividades- y metodológicos - la construcción de series, el análisis contrastivo de textos y la reformulación. En todos los casos partiré de trabajos de investigación que he realizado, que me permitirán introducir e ilustrar los diferentes temas. Si bien se refieren al ámbito hispánico en muchos casos son próximos a otros espacios culturales.

\section{Glotopolítica e Historiografía lingüística}

En la regulación de la lengua y los discursos, las gramáticas y los textos que se inscriben en el amplio campo de la retórica (como, entre otros, manuales de estilo, artes de escribir, tratados de correspondencia) han tenido un papel fundamental y se han multiplicado en las lenguas vernáculas europeas desde el Renacimiento. Las gramáticas, fundamentalmente, han sido objeto de estudio de la Historiografía Lingüística ya que esta "tiene como objeto la historia de la lingüística", es decir, "trata de describir, interpretar y explicar (segmentos de) la historia de la lingüística" (SWIGGERS, 2009, p. 69-70) o, en términos más amplios, de describir "los principios y métodos de la producción del conocimiento lingüístico y de sus resultados en un determinado momento, inevitablemente histórico" (ALTMAN, 2009, p. 128). Aunque algunas de sus expresiones no dejan de considerar la relación de los textos con los datos contextuales, la Historiografía Lingüística se interesa, en particular, por el proceso que va conformando la disciplina a través de los modos de conceptualizar, la delimitación de categorías, las reformulaciones didácticas, las maneras de abordar problemáticas teóricas (la creación léxica o el origen del lenguaje) o aspectos como la actitud frente a la norma o las cualidades de las lenguas.

Si bien la Glotopolítica cuando analiza instrumentos lingüísticos no es ajena a las consideraciones de la Historiografía Lingúistica sobre ellos, focaliza esos textos, como lo he venido señalando, como intervenciones sociales, asociadas a ideologías lingüísticas, destinadas a influir en las prácticas de una comunidad, más o menos amplia, tendiendo a modificar el espacio público del lenguaje, sujetas a requerimientos de la sociedad frente a los cuales se adoptan, además, determinados posiciona- 
mientos. De allí el interés por el análisis de la articulación de los textos con sus condiciones de producción y de circulación. No abordamos los instrumentos lingüísticos primariamente por su posible ubicación en una Historia de la Lingüística o, incluso, en una más amplia Historia de las Ideas sobre el Lenguaje sino por su inscripción en los procesos históricos y en especial en lo que corresponde a su dimensión política. Al analizarlos interrogamos también los modos de conceptualizar, el juego de las categorías, las tradiciones lingüísticas o los otros aspectos que considera la Historiografía Lingüística - como la atmósfera intelectual o la institucionalización de la reflexión lingüística en determinados períodos (BATISTA, 2013, p. 63-64); o la comprensión de la dinámica de las redes sociales (KOERNER, 2007, p. 40) y atendemos al hecho de que la mayoría de estos instrumentos lingüísticos están vinculados con experiencias pedagógicas o surgen en relación con la puesta en marcha o avances en el sistema educativo. Pero esos instrumentos nos interesan fundamentalmente como indicios de transformaciones en curso o de toma de posición de un sector social, como formas de reproducir la estructura de clases dominante o como expresión de una voluntad de cambio. De allí la importancia del abordaje discursivo de los materiales que nos va a permitir reconocer regularidades, identificar asociaciones, atender a fenómenos periféricos pero significativos que orienten en ese sentido la interpretación.

\section{Temporalidades y espacios}

Cuando hacemos el recorrido de la constitución del campo de la Glotopolítica en el mundo académico lo ubicamos después de la Segunda Guerra Mundial, tal vez porque es el momento en el que comienza el proceso que llevará a una economía - mundo planetaria y que permite, con su relativa distancia, interrogar la zona anterior, la de conformación de los Estados nacionales. Nuestros trabajos atienden, en general, a este largo proceso, el de formación y desarrollo de los Estados modernos, y a las transformaciones operadas por el de la globalización. En esos marcos analizamos fenómenos que se inscriben en diferentes temporalidades. Muchos historiadores siguiendo a Fernand Braudel (1979) hablan de la larga duración, la duración media, la corta duración, a lo que podemos agregar acentuando la focalización el acontecimiento como cristalización en un determinado tiempo y lugar de un conjunto de líneas de fuerza. Por supuesto que son todas medidas relativas que dependen de la relación que se establezca con 
otros fenómenos en un determinado campo y de lo que quiera interrogar el investigador.

En relación con el núcleo central de la exposición -los instrumentos lingüísticos y, en especial, las gramáticas y retóricas- podemos decir que nos ubicamos en la larga duración cuando analizamos los tipos de gramáticas - particulares, de Estado y generales- que se produjeron en Europa desde el Renacimiento hasta el siglo XIX (ARNOUX, 2008) y que correspondieron a las diferentes fuerzas que en el marco de la economía mundo occidental fueron conformando los Estados nacionales: esquemáticamente, la que tendía a multiplicar los intercambios en esa vasta zona (las generales), la que centralizaba jurídica y administrativamente el poder (las de Estado) y la que recorría el territorio buscando instaurar también un mercado nacional (las particulares). Este ciclo se cierra con las gramáticas nacionales, que sostienen el imaginario de lengua común, cuya expresión más acabada en el ámbito hispánico es la Gramática castellana destinada al uso de los americanos (Chile, 1847) de Andrés Bello que consagra la variedad que va a circular por el Estado, definiendo a la vez los modos legítimos y marginalizando los otros.

Nos ubicamos en una duración media cuando estudiamos la serie de retóricas que desde la primera década revolucionaria (la de 1810 en el Río de la Plata) hasta principios del último tercio del siglo XIX se produjeron, particularmente en lo que va a ser el territorio argentino, y que formaron a la elite criolla que debía encarar las nuevas tareas estatales posteriores a la Independencia (ARNOUX, 2013c). A esta etapa la clausura de la expansión de la escuela secundaria que lleva a que la enseñanza retórica se restrinja notablemente en relación con los nuevos sectores sociales que ingresan y que la clase dirigente aprenda la discursividad pública en otros espacios de socialización o en los estudios superiores. Se pasa así, por ejemplo, en los manuales escolares, del reconocimiento de los variados géneros ligados a las prácticas sociales (discursos ministeriales, arengas, artículos periodísticos, textos de historia, discursos de homenaje a los próceres, respuestas a interpelaciones del congreso), necesarios para la formación de la elite en la primera etapa de conformación del Estado, a los géneros escolares que privilegian o géneros breves como la carta o secuencias desligadas de los géneros mayores como el retrato, a la vez que en la enseñanza media se va afianzando la historia de la literatura. Este nivel no va a formar ya a la clase dirigente para su desempeño en funciones altas del aparato estatal sino que va a tender a una enseñanza general 
y de tránsito a los estudios superiores en el bachillerato o con cierto grado de especialización para funciones sociales definidas en las escuelas de comercio, las normales o los colegios industriales.

Focalizamos una duración más breve cuando contrastamos las gramáticas castellanas (llamadas "compendios", en general) para el ciclo medio del sistema educativo que se publican en la década del cincuenta del siglo XIX, antes de la centralización estatal de la escuela secundaria que se va a dar en la segunda mitad (ARNOUX, 2014b). Al atender a la sintaxis, por ejemplo, se evidencia la tensión entre el saber gramatical y la representación, todavía no claramente establecida, del sujeto que va a ser escolarizado en ese nivel, de allí las vacilaciones acerca del alcance y tratamiento de esta zona de la gramática. Pero también aparecen diversas orientaciones, en las que conviven viejos temas con las tendencias que van a imponerse. Esto nos permite reconocer cuando analizamos las gramáticas de esa década el paso de una gramática de la proposición a otra de la oración que atiende a la actitud del hablante, el avance de otros tipos de subordinación más allá de la proposición relativa, la insistencia en la relación entre la función y las clases de palabras, la progresiva aceptación de los marcos mayores para el análisis sintáctico. A la vez, en esta zona de la gramática más que en otras, avanza la preocupación por que la enseñanza gramatical sirva para enseñar a pensar realizando operaciones "racionales" que puedan proyectarse al ámbito social acompañando la organización y modernización del Estado.

Y, finalmente, abordamos el acontecimiento (acontecimiento discursivo, en este caso, en términos de Guimarães, 1995) cuando estudiamos los dos tomos de la Gramática castellana de Amado Alonso y Pedro Henríquez Ureña, publicados en 1938 y 1939, textos fundadores de una tradición en la enseñanza de la lengua en la Argentina. En ellos el cerrado dispositivo textual tiende al disciplinamento integral de los sujetos en un momento de marcada heterogeneidad de origen en la población de los grandes centros urbanos de la Argentina, particularmente Buenos Aires (ARNOUX, 2001b). En el texto se evidencia la preocupación por poner orden en el pensamiento gramatical escolar y en las actividades de lectura y escritura y por controlar el despliegue del intelecto y la afectividad. Al mismo tiempo, se muestra la voluntad de incidir en la oralidad como una forma de luchar contra la anarquía generada por la ausencia de un centro normativo aceptado, tema que aflige particularmente a Amado Alonso quien se había referido a ello en varios trabajos. Disciplinar la lengua es también disciplinar la sociedad. 
Si bien en la interpretación atendemos al despliegue en una temporalidad, no está ausente la consideración de las otras, que conforman los necesarios marcos interpretativos o las capas de memoria que pueden manifestarse de distintas maneras. Si atendemos a la Gramática castellana de Andrés Bello, en su particularidad, no podemos dejar de considerar la larga duración en la que se inscribe. El peso de las gramáticas de Estado aparece en la fuerte vocación normativa; el de las particulares se evidencia en su preocupación por recorrer el territorio de las variedades legítimas apelando a un corpus importante de muestras de lengua; las gramáticas generales inciden en la preocupación por dar razón de los hechos del lenguaje y aplicar la racionalidad moderna al análisis de los fenómenos lingüísticos. Así, al mismo tiempo que sienta las bases de una gramática nacional adecuada a los nuevos Estados y, por lo tanto, atenta al sistema de la lengua y a la unificación y explicitación de los criterios de análisis, se define respecto de la tradición y hace las opciones teóricas y metodológicas que considera adecuadas en esa etapa. Reconocer el diálogo con esas tradiciones discursivas nos permite comprender mejor las posiciones que adopta en la elaboración del texto gramatical.

En cuanto a los espacios que consideramos, ellos no son solo el estatal sino también aquellos con los que de distinta manera se interactúa en una u otra etapa: lo local, lo regional y lo global. Lo primero, es decir lo localmente situado, lo debemos tener en cuenta si nos interesamos, por ejemplo, por la producción de los profesores del Colegio Monserrat de Córdoba (que dependía de la primera universidad que se estableció en lo que sería luego el territorio argentino) cuando esta institución pasa, en la segunda mitad del siglo XIX, a integrar la red de los colegios nacionales. Los docentes tuvieron que preparar material didáctico destinado a responder a los nuevos programas. Esos emprendimientos no podían dejar de considerar el peso de la tradición escolar propia, en la que la religión ocupaba un lugar no desdeñable. Estas respuestas locales a decisiones del Estado nacional dan lugar a tensiones que los mismos textos exponen y cuyo análisis exige atender al contexto inmediato.

Consideramos lo regional, cuando discutimos sobre el carácter de los instrumentos lingüísticos que deben acompañar el proceso de integración regional sudamericana y evaluamos los existentes atendiendo a la valoración de las variedades hispanoamericanas y a la necesaria apertura al área lusófona. También enfocamos ese espacio cuando analizamos los instrumentos lingüísticos producidos en el marco de las políticas lingüís- 
ticas de área idiomática que se asientan en una declarada gestión democrática de la lengua compartida (ARNOUX, 2013a), aunque sea marcado el peso del país que encabeza el área por su poder económico (España, en el caso hispanófono, por ser parte de la Unión Europea). Aquel principio lleva a proponer una norma pluricéntrica que, a la vez, tenga en cuenta los aspectos convergentes que permiten hablar, en el caso del español, de una lengua general para el área.

Abordamos lo global cuando nos centramos, dentro de la política lingüística panhispánica, en las orientaciones destinadas al periodismo en línea que aparecen en los nuevos manuales de estilo para el sector, que buscan imponer un español "global" y una discursividad que llegue a "todo el mundo" (ARNOUX, 2015b). Para los medios digitales se piensa en una lengua "neutra", que limite los localismos y en una prosa informativa que evite los juegos de lenguaje, como las metáforas, y en la que domine la brevedad y la economía de recursos. "La unidad en la diversidad", propia de las políticas de área idiomática, deja paso a la uniformidad que se asocia con los nuevos medios. Se piensa que esto va a facilitar no solo la acción de los buscadores sino también la expansión planetaria del español y la articulación con el inglés ya que comparten el ideal de prosa informativa "digital".

\section{Los instrumentos lingüísticos y la importancia del intertexto}

El sintagma, instrumentos lingüísticos, fue acuñado por Sylvain Auroux (1994) para referirse, en principio, a los que considera los dos pilares del saber metalingüístico, las gramáticas y los diccionarios. Insiste en "su carácter de artefactos: existen como objetos técnicos", "disponibles entre la comunidad como medios para encontrar una forma, referencias y normas que ninguno de los locutores posee integralmente" (AUROUX, 2009, p. 141-142). Su alcance se amplía a todos aquellos que exponen un saber metalingüístico que puede ser tanto de naturaleza especulativa como práctica. En este último caso se refiere a aquellos cuya meta es adquirir un dominio ya sea de la enunciación (la capacidad del hablante de adecuar sus palabras a un objetivo dado) de lenguas (materna u otras) o de la escritura (la capacidad de leer y escribir (AUROUX, 1989, p. 18). Podemos incluir, así, entre otros, tratados sobre aspectos del léxico, glosarios, silabarios, diccionarios bilingües y de sinónimos, manuales de traducción, ortografías, comentarios sobre el estilo de los autores, retó- 
ricas, artes de escribir, manuales de correspondencia, manuales de estilo periodísticos, artes de predicar.

Estos textos dialogan por cierto con otros que hay que considerar cuando el objetivo de la investigación lo requiera. Así, puede ser necesario interrogar ensayos sobre la lengua, notas normativas en los periódicos, notas lingüísticas a textos clásicos, antologías escolares, obras referidas a aspectos teóricos, reseñas, boletines académicos, programas escolares, traducciones, resoluciones de política lingüística, prólogos a ediciones de textos literarios, discursos de creación o apertura de instituciones dedicadas a las lenguas y sus posibles estatutos.

Por ejemplo, en la primera década revolucionaria, en 1817, aparecen en Buenos Aires dos gramáticas escolares, una de Antonio José Valdés, cubano, y otra de Felipe Senillosa (ARNOUX, 2012; 2013b), ambos además dirigen periódicos, lo que muestra la vinculación entre la expansión de la prensa gráfica y el desarrollo de gramáticas dirigidas a un público amplio aunque se presenten en principio como escolares. En sus respectivos periódicos escribe cada uno una nota gramatical. Analizarlas permite entrever el alcance que asignan a la norma: en Valdés predomina la regulación gramatical y léxica destinada a la unidad del mundo hispánico sostenida por la Real Academia Española (RAE) aunque hable de una "gramática patria"; en Senillosa, la preocupación reside en la combinación de las palabras en el sintagma y la regulación a la que tiende es la del sentido, pero al hacerlo muestra en los ejemplos (que corresponden a términos con valores semánticos inestables en ese momento: patria, país, suelo) cómo esto no puede resolverse desde la gramática sino desde la política. Las notas permiten apreciar aspectos no dichos en las gramáticas pero que nos ayudan a comprender, a partir de una reflexión marginal sobre la lengua, los respectivos posicionamientos en una época turbulenta y la representación que tienen de la función social del pensamiento gramatical y del sentido de su enseñanza (ARNOUX, 2011). En Valdés se impone una perspectiva normativa dependiente de la Academia y cierto dogmatismo en la exposición gramatical y en Senillosa una preocupación por atender a los significados y por reflexionar razonadamente acerca de los fenómenos gramaticales sin atenerse a las posiciones de la RAE, a la que por otra parte cuestiona.

En algunos casos, pueden considerarse, incluso, textos que no tematizan el lenguaje pero que construyen representaciones de lo social que permiten comprender las tensiones, las limitaciones, los objetos pri- 
vilegiados por los textos lingüísticos, las exclusiones que operan, incluso las decisiones teóricas que adoptan.

En la etapa de conformación de los Estados nacionales hispanoamericanos, los gestos glotopolíticos se asientan, por un lado, en la memoria de la Independencia que implicaba un corte con España que debía marcarse en la lengua y, al mismo tiempo, un vínculo fraterno con los países hermanos. Estos movimientos se asociaban en el plano lingüístico con la representación del español americano como lengua compartida. Diversos textos que no tematizan el lenguaje remiten a ese imaginario de la unidad hispanoamericana. Sin embargo, a mediados del siglo XIX se están conformando los nuevos Estados que necesitan ir construyendo un imaginario propio que articule territorio, lengua y raza. Los manuales de historia colaboran en ello y van a sostener a su manera la representación de lengua nacional asociada al Estado. En Chile, por ejemplo, el Manual de Istoria de Chile (1846) de Vicente Fidel López cumple esa función. Es un texto para la escuela primaria revisado y aprobado por la Universidad, que excluye de la nación chilena a los indígenas porque son de otra raza y, como manifestación de ello, no hablan el español. Esta representación de nación restringida, ligada a la organización de los nuevos Estados no anula la más amplia, aunque quede en una posición subalterna, de nación hispanoamericana asociada con la memoria independentista en la cual la comunidad de lengua era un factor importante. La tensión entre estas dos representaciones, que en cierta medida compiten en esa etapa, explica los alcances o las limitaciones de algunos gestos glotopolíticos.

Un caso interesante es el de la reforma ortográfica chilena aprobada por la Universidad de Chile en 1844. Implicó un gesto estatal enérgico de marcar la nación en la lengua, lo que solo se podía hacer desde esa instancia, pero al mismo tiempo el horizonte de expectativas era el español americano. El desajuste entre una intervención que comprometía solo al Estado chileno y la representación más amplia de un espacio común asentado en una lengua compartida explica las vacilaciones primeras y el abandono posterior de la reforma. Tanto el texto histórico que recortaba la nación como otros que muestran la presencia de un imaginario nacional más amplio, hispanoamericano o sudamericano, constituyen un apoyo inestimable para comprender lo que se juega en campo del lenguaje, incluso en el texto más importante del período que es la Gramática Castellana de Andrés Bello.

Bello, que es sensible además a la unidad del mundo hispánico 
(propio de una posición más conservadora en el campo social), busca en el texto gramatical resolver las tensiones entre estas diferentes representaciones (ARNOUX, 2008). Por un lado, en el paratexto (título y prólogo) privilegia el espacio hispanoamericano. Por otro lado, en los ejemplos, que en muchos casos manipula, establece la continuidad entre el español peninsular y el americano. Y, finalmente, proyecta en las opciones teóricas y metodológicas de la gramática su representación del Estado nacional con sus atributos de autonomía, centralización, control territorial, racionalidad, legitimidad. Consagra, así, la autonomía de la lengua, rechazando las remisiones a otras, incluso al latín. Pone en marcha un complejo dispositivo normativo que define la norma como el uso de la gente educada. Se atiene a la superficie gramatical sin recurrir a explicaciones que deban apelar a fenómenos como la elipsis. Insiste en la utilización de criterios simples y uniformes para todos los casos, es decir, aplica la racionalidad moderna. Acepta, finalmente, la igualdad formal de algunas variedades al mismo tiempo que desconoce o marginaliza otras. Asocia, así, de una manera original la lengua al Estado nacional, apoyándolo a partir de la unidad de la lengua y del saber sobre ella (ORLANDI, 2000). Pero a la vez declara su voluntad hispanoamericana y no desdeña la unidad lingüística del mundo hispánico. Estos tres aspectos de su obra, que implican alcances diferentes de la "lengua nacional", se explican por las distintas representaciones que circulan y que podemos relevar en textos de la época.

\section{El dispositivo normativo y su función social}

A partir del hecho de que los instrumentos lingüísticos describen y prescriben al mismo tiempo, Louis Queré (1987, p. 67-76) señala, en la línea de Pierre Bourdieu (1988 [1987]), que

la constitución de un corpus de descripciones y de explicaciones de las prácticas lingüísticas en términos de reglas, de máximas, de definiciones, de sentidos estables atribuidos a las palabras, de cosas que se pueden decir o no decir, de usos correctos o inaceptables, de desvíos típicos, etc. [...] opera como instrumento de normalización de las prácticas, es decir, de determinación de su cumplimiento por un principio de orden o una "forma normal" enteramente exógenas (articulación y traducción propia).

Estos instrumentos no solo exponen un saber destinado a incidir en las prácticas comunicativas disciplinándolas sino que también establecen 
jerarquías entre aquellos sometidos a las normas y los que son ajenos o se desvían de ellas. El Epítome (RAE, 1857), la gramática escolar derivada de la gramática académica de 1854, por ejemplo, advierte a los alumnos, mostrando el corte entre ellos, que van a aprender la gramática, y los otros que por el desconocimiento de ese saber hablan mal:

El que no sabe gramática habla con desaliño, usa impropiamente las palabras (que también se llaman términos, voces, vocablos y dicciones), las coloca sin orden y las pronuncia indebidamente: de donde resulta que los que le escuchan le entienden mal o no le comprenden. No hay mayor rudeza que el hablar mal, es decir, sin observar las reglas de la gramática; y como el que no la sabe no conoce bien su lengua, tampoco puede entender bien lo que oye o lee, ni por consiguiente adelantar en otros estudios para cultivar su entendimiento (RAE, 1857,p. 24).

Se sigue así una extensa tradición en los textos normativos en la que las diferencias sociales se explican por el desconocimiento formal de los dispositivos y no por los lugares sociales en los que están ubicados los hablantes.

Michel Foucault se ha detenido en la importancia de la norma en las sociedades occidentales modernas y su función como criterio de división de los individuos ya que genera una clasificación, una jerarquización, calificaciones, el establecimiento de límites, y exige el diagnóstico (FOUCAULT, 1994, p. 75-76). Edgardo Castro (2011, p. 282) sintetiza la concepción de la norma en Foucault planteando que esta "remite los actos y las conductas de los individuos a un dominio que es, a la vez, un campo de comparación, de diferenciación y de regla a seguir (la media de las conductas y los comportamientos)"; que "diferencia a los individuos respecto de este dominio considerado como un umbral, una media, un optimum que hay que alcanzar"; que "jerarquiza en términos de valor las capacidades de los individuos"; y que "impone una conformidad que debe alcanzarse, [es decir] busca homogeneizar". Los instrumentos lingüísticos responden, en mayor o menor medida, a esos rasgos.

Aquellos que han dominado, fundamentalmente hasta la acentuación del proceso de globalización, son dispositivos normativos que se deslizan de "lo normal" (la media) a lo prescriptivo. En algunos casos autorizan la condena porque señalan lo prohibido ubicándose en la ley más que en la norma. Pero este aspecto ha tendido a borrarse del discurso gramatical así como este se abre en la actualidad a usos alternantes aunque restringidos a la variable geográfica (las formas cultas de las distintas 
zonas). Prueba de lo primero son las diversas fórmulas que señalan lo cuestionado en la Gramática Básica de la Lengua Española (2011), tercer ejemplar de las gramáticas de la Real Academia Española destinado a los niveles primeros de enseñanza o al gran público, que aunque use el "no se dice" o "no se considera correcto" va a recorrer un amplio espectro: entre otros, "se recomienda evitar", "algunos admiten pero se prefiere la otra opción", "si bien, sobre todo en publicidad, es frecuente, es una opción que no se recomienda", "su aceptación es desigual en los distintos países", y en algunos casos "se admite la variación".

Estas fórmulas, aunque débilmente permisivas, revelan también la acción, por supuesto muy atenuada, de lo políticamente aceptado en los instrumentos lingüísticos postmodernos: no hay que imponer sino aconsejar. Pero en realidad son leídas en el molde clásico de lo correcto o incorrecto porque es lo que se espera de los segmentos normativos enunciados por la autoridad académica y, sobre todo, porque los ejemplos son construidos y, por lo tanto, desprendidos de un posible entorno discursivo, lo que hace difícil su cuestionamiento. A eso se agregan fenómenos generadores de inseguridad lingüística. Por un lado, los hablantes se encuentran con expresiones no recomendadas que forman parte de su abanico expresivo o de lo que percibe como norma social en su comunidad, ya que se rechazan, por ejemplo, formas del lenguaje político corrientes en personajes prestigiosos. Por el otro, las valoraciones atienden a diversos criterios que se emplean erráticamente según las formas (frecuencia, juicios de valor de hablantes privilegiados, criterios gramaticales, de distribución geográfica, regularidades reconocidas...). Y, finalmente, se acentúa la dogmatización del pensamiento gramatical ya que no se suministran explicaciones en los textos destinados al gran público (ARNOUX, 2015a).

Así, a pesar de las concesiones de época, estos tramos de la gramática académica destinada a "todo el mundo" cumplen la función de subalternización de aquellos hablantes cuya variedad es diferente de la preferida o que no pueden relativizar la norma contextualizando los ejemplos. Esta subalternización, que recurre a distintos procedimientos según los textos y las épocas es una de las formas de reproducir las desigualdades sociales, en lo que los instrumentos lingüísticos en muchos casos han colaborado. 


\section{La construcción de subjetividades}

Señalábamos al comienzo que las intervenciones sobre el lenguaje son también operaciones que participan en la conformación de las subjetividades que la sociedad requiere. En este punto debemos recordar que las subjetividades son morosas y que, como señalamos también al referirnos a las temporalidades, en ellas no están ausentes elementos asociados con otras memorias. Deleuze (2015 [1986], p. 138) comentando a Foucault, dice "las más viejas subjetivaciones, es decir las maneras de constituirse el sujeto [...], por más completamente inadaptadas que sean continúan trabajándonos". De allí que en el análisis de materiales o situaciones nos podemos encontrar con estratos de representaciones correspondientes a distintas temporalidades y debamos interrogarnos por qué se activan e, incluso, por qué se activan diferentemente según las posiciones o los posicionamientos sociales.

En el Congreso Internacional de la Lengua Española de Panamá, en la mesa destinada a los nuevos medios, por ejemplo, una de las ponentes del panel sobre el periodismo y los medios digitales, Olga Olbadía de Díaz, exaltaba la horizontalidad de la comunicación, la interactividad, el uso de un español global, de una discursividad que simplifica y sintetiza, apelando a las representaciones elogiosas, comunes de esta época, acerca de lo digital. Pero, a la vez, cuestionaba uno de sus resultados, el lenguaje utilizado en la red por los usuarios (que comentaban, con la visibilidad propia del medio, las noticias), por su desdén de la norma: entre otras, la puntuación incorrecta, los neologismos mal utilizados, la abundancia de abreviaturas, la aversión a la tilde, ubicándose en una posición normativa tributaria de ideologías lingüísticas anteriores. ¿Por qué se activa? Por un lado, por lo que dijimos antes respecto de que las subjetividades son morosas y las ideologías lingüísticas anteriores sobreviven y pugnan por manifestarse. Pero, por otro lado, porque hay una necesidad presente que las activa y les da sentido. En este caso, posiblemente se active la crítica normativa porque una cierta uniformidad es necesaria para homogeneizar el lenguaje en la red y facilitar las búsquedas de datos. Por supuesto que el problema no va a residir en las tildes ni en el uso inadecuado de mayúsculas o minúsculas ni en la puntuación errática, que se resuelven con mayor facilidad, pero tal vez sí si se afecta la separación de palabras (salvo cuando tengan la marca de etiquetas) o si la escritura adopta formas difíciles de genera- 
lizar. Aunque es posible que el avance tecnológico solucione también estas dificultades.

Estas representaciones heterogéneas del ejemplo anterior exponen en el nivel del lenguaje el hecho de que la sociedad industrial (asociada con el disciplinamiento, que fija el cuerpo individual y social en un conjunto de normas, y con la regularización de la vida o lo que Foucault, 2014, llamaba la Biopolítica) convive con otra, postindustrial, en la que reina, como esbozamos antes, la ilusión de libertad generada por los medios digitales. En ellos los sujetos circulan y nutren los Big Data que permitirán no solo vigilarlos sino ubicarlos en nuevas clasificaciones que orientarán la acción política o la comercial (lo que varios estudiosos, entre otros Han, 2014, llaman la Psicopolítica que no gestiona los cuerpos, como la Biopolítica, sino las "almas", haciendo visibles y produciendo modelos de comportamiento colectivos).

Para estimular el espíritu de libertad y facilitar la circulación en la red, para hacer amigables y seductores los medios digitales, para acercar lo lúdico a la escritura, todo despliegue lingüístico es admitido y legitimado pero este tiene que ir esquematizándose (como los emoticones) y, como dijimos, uniformizándose para perturbar lo menos posible la acción de esos buscadores. Como dice Han (2014, p. 121) en "el infierno de lo igual alcanza la comunicación su velocidad máxima". Los Manuales para la escritura en Internet registran y prescriben, en ese sentido, los usos más convenientes. A la brevedad y claridad, agregan, como señalamos antes, la ausencia de localismos, la guerra a las metáforas, pero también el apoyo a la comprensión de la ironía con recursos gráficos (estiramientos) u onomatopeyas, la simplicidad ya que la representación del lector es de alguien con escasos mecanismos inferenciales, el despliegue icónico de la emotividad o el uso de segmentos fijos: "me gusta".

Mario Tascón, en Escribir en Internet (FONDÉU, 2012, p. 39), por un lado, justifica en la lógica del software las transgresiones normativas, que en los textos académicos eran condenados: "Esta forma de escribir (los números) que la Real Academia recomienda choca con la lógica del software y hace que quien la emplee se encuentre luego con problemas para utilizar su material. Todo por motivos que van más allá de los argumentos clásicos y que tienen que ver con la tecnología". Por otro lado, apela al imperativo de la visibilidad total: "También está la necesidad de que los textos que escribimos sean 'comprendidos' por las máquinas y sus algoritmos para que, a través de plataformas como son los buscadores 
otras personas puedan encontrarnos" (el resalte me pertenece). Además anuncia con entusiasmo que la máquina escriba por nosotros:

Veremos también frases, incluso párrafos, en los que algunos verbos, adjetivos y sustantivos serán "escritos" por las máquinas a medida que las reglas que las manejan tengan determinadas entradas de datos. No vamos a esperar de momento calidad literaria, pero seguro que aumentará la eficacia de la comunicación, sobre todo en campos como la economía o el mundo de la información. (FONDÉU, 2012, p. 40)

Estos textos muestran cambios en los dispositivos normativos y nuevas representaciones de sujeto que son aceptados con entusiasmo por los mismos manuales, en este caso de la Fundación del Español Urgente ligada tradicionalmente a la Real Academia, porque se presentan como sostenidos en prácticas espontáneas de los usuarios o en requerimientos de la comunicación contemporánea a los que se someten sin discusión. El nuevo disciplinamiento al que se tiende resulta más eficaz que los anteriores porque es asumido y reproducido por los sujetos como ejercicio de la libertad y la creatividad.

\section{El cotejo de textos: series textuales y análisis contrastivo}

Cuando abordamos los instrumentos lingüísticos no deja de llamar la atención la estabilidad discursiva de los textos, que se manifiesta en el plan textual, en los formatos dominantes, en las categorías que consideran, en la inclusión de los ejemplos, en las operaciones que se solicitan del lector, en los modos de prescribir. Por eso desde la perspectiva glotopolítica resulta productiva la construcción de series de textos (SCHLIEBEN-LANGE, 2000), como se mostró ya en algunos ejemplos, a partir de las hipótesis de trabajo que se formula el investigador, o estudiar series que de alguna manera ya están establecidas como la de la gramática académica y sus dos versiones para un público más amplio o para distintos niveles de enseñanza. En el primer caso, se puede llegar a hacer un corte sincrónico como propusimos en relación con las gramáticas para la escuela secundaria, o convocar la media duración, como en el caso de las retóricas, o la larga duración, como en las gramáticas desde el Renacimiento hasta el siglo XIX. Los ejemplares que integran las series pueden corresponder a un espacio nacional, regional o cultural amplio, a una misma lengua o a varias. Estas series permiten en la comparación de 
aspectos equivalentes reconocer las diferencias, que muestran las tensiones entre memorias discusivas o entre ellas y los nuevos requerimientos sociales, o definir posicionamientos respecto de los mismos fenómenos o de fenómenos próximos.

Cuando abordamos el tratamiento de la elocuencia en la serie de las retóricas del ámbito hispánico en la primera mitad del XIX (ARNOUX, 2015c), destinadas a formar en la escuela secundaria a los nuevos sectores de las clases dirigentes, se evidencia en el contraste de los ejemplares las necesidades del momento y los procesos en marcha. Así, la mayoría los textos se ven obligados a actualizar los rasgos de estilo en virtud de las nuevas prácticas, lo que hacen a partir de marcar las diferencias entre la elocuencia de los antiguos - más cercana a lo que rechazan, la de las épocas tumultuosas - y la de los modernos que no se dirigen al pueblo sino a sus pares en ámbitos deliberativos, lo que impone evitar los desbordes y asignar al texto un mayor desarrollo argumentativo a la vez que se insiste en la formación de la moral cívica. Algunos integran en la elocuencia el ejercicio del periodismo, aunque sea una manifestación escrita, o de la clase en las instituciones de enseñanza superior. Esto lleva a plantear la importancia de otros conocimientos más allá del retórico. En relación con la actio se considera, posiblemente por la incidencia del Romanticismo, la inspiración en el ejercicio de la palabra pública y, en relación con esta, se pasa de la preocupación por una preparación previa a dejar fluir la subjetividad. En los modelos se muestra el tránsito de los latinos, a los europeos y a los nacionales privilegiando cada vez más las expresiones literarias y, en el dispositivo normativo, una mayor preocupación por fundamentar las reglas. De las opciones que las retóricas exponen se deriva la representación que tienen en esa etapa de los miembros de la clase dirigente, que deben presentarse como cívicamente virtuosos, estar en condiciones de controlar las emociones, desempeñarse con elocuencia en los espacios deliberativos y si es necesario en las instituciones educativas, desplazarse a la escritura en la prensa, tener un conocimiento de los campos científicos de la época y ser capaz de apelar a modelos en la lengua nacional. La serie nos permite también apreciar lo que va erosionando la tradición retórica: el rechazo a las reglas, la importancia creciente de la literatura y la valoración de otros conocimientos como centrales para el desempeño público.

En algunos casos va a ser más interesante el contraste de dos textos. Estos pueden ser muy próximos temporalmente como las gramáticas de Valdés y Senillosa a las que nos referimos que fueron publicadas en 1817 
y que se inscriben en corrientes gramaticales diferentes. $\mathrm{O}$ estar separadas temporalmente como en el caso del Diálogo de la lengua de Juan de Valdés y el Arte Grande de la Lengua Castellana de Gonzalo Correas, escrito uno en 1535 y 1536 y el otro en 1626 . Son estos dos textos representativos de las gramáticas particulares renacentistas cuyas cercanías son significativas, que recorren las variedades, muestran las dificultades de establecer una norma, señalan relaciones con otras lenguas, definen una fuerte figura de autor, y optan por un despliegue discursivo que, en mayor o menor medida, se diferencia del texto consagrado de Nebrija (ARNOUX, 2013d).

Cuando un texto deriva de otro, el contraste nos permite analizar las operaciones de reformulación.

\section{La reformulación}

Es habitual en los textos normativos la reformulación de textos anteriores del mismo autor o de otros, en la misma lengua o en otra, de la totalidad del texto o del dispositivo global de la obra o de partes o de secuencias como los ejemplos, las definiciones, las reglas, las notas, los cuadros y también los prólogos, las presentaciones o las advertencias. Las modalidades pueden ser diversas: entre otras, resumen, expansión, adaptación didáctica, traducción, apropiación de estrategias expositivas. Cuando los datos contextuales, las indicaciones paratextuales o el trabajo detenido sobre los materiales muestra que un texto deriva total o parcialmente de otro, el estudio de las operaciones de reescritura (omisiones, expansiones, desplazamientos, sustituciones) nos permite entrever las representaciones de género, de destinatario, de la nueva situación de enunciación, del ámbito de circulación posible del nuevo texto, de las transformaciones operadas en la reflexión sobre el lenguaje, de aquello que por diferentes razones no puede más ser dicho o no puede ser dicho en determinadas circunstancias, de las opciones teóricas privilegiadas, del alcance de las filiaciones reconocidas explícitamente o no y de las exclusiones.

Un caso interesante que surge del cotejo de textos son las omisiones que el prólogo de la gramática académica de 1858 hace del de 1854 (ARNOUX, 2015d), aunque se presente como un simple retome indicado además por las comillas. Una de ellas es la supresión de los segmentos en los cuales se vincula la actividad gramatical con otras prácticas. Cuando discute la necesidad de cambios de nombre de las categorías, la gramática de 1854 dice: 
[...] el pintor y el herrero que se empeñaran en llamar de otra suerte al pincel y a la lima no manejarían con mayor facilidad esos dos instrumentos a favor de la mudanza de nombre (p.5).

Y afirma luego el uso de la nomenclatura tradicional en el hecho de que son tecnicismos que se usan en una acepción particular, propia del saber gramatical y muy alejada del significado corriente del término:

Los primeros gramáticos que la usaron [a la palabra analogía] estuvieron en su derecho dándole aquel nuevo significado, como hizo el que aplicó a la arquitectura militar la palabra cortina, que tiene allí muy diverso valor que en el uso doméstico (p. 6)

Esta supresión de la posible proximidad de la actividad gramatical con otras prácticas sociales se explica por la acentuación de una actitud conservadora por parte de la Academia que debe mostrar su distancia, particularmente en una época en la que los sectores progresistas, que habían puesto en marcha una serie de reformas, son desplazados del poder. Así, la simple supresión de dos fragmentos nos lleva a interrogar el marco histórico en el que el texto se ha producido.

La reformulación puede implicar actividades de traducción. Pero aquí también el contraste del texto fuente y el texto meta nos permite reconocer aquello que no puede ser dicho en la nueva situación de enunciación. Así, cuando nos centramos en la parte gramatical del Curso de Humanidades Castellanas escrito por Jovellanos, para el Real Instituto de Náutica y de Mineralogía de Gijón, entre 1794 y 1797, en la que reformula la Grammaire de Condillac y contrastamos ambos textos (ARNOUX, 2001a; 2003) se evidencian en las omisiones las dificultades que pesaban sobre la minoría ilustrada española en esa etapa. Jovellanos, a la vez que parte de una de las formulaciones más avanzadas del pensamiento europeo sobre el lenguaje, debe responder al peso de la tradición eclesiástica en el aparato monárquico cuyo apoyo necesita, lo que lo lleva en ciertas zonas del texto a olvidar la fuente. De allí que prime una concepción memorística en el aprendizaje, que elude discursivamente en muchos tramos el despliegue razonado; que borre lo referido a la génesis del lenguaje, central en Condillac que plantea un primer lenguaje de "acción" sostenido en gestos, movimientos del rostro y sonidos no articulados; y que desplace el Análisis del Discurso de la posición inicial que tiene en la fuente como modo de presentar cómo las lenguas analizan el pensamiento, a la parte anterior a las gramáticas en inglés y francés. 


\section{Conclusión}

A través de la exposición he querido mostrar, a partir de ejemplos variados, cómo la Glotopolítica aborda los instrumentos lingüísticos. El estudio de los modos de representar, por ejemplo, el saber gramatical o retórico nos permite gracias al trabajo con series, el análisis contrastivo de textos, la consideración de las operaciones de reformulación o el trabajo detenido sobre zonas, equivalentes formalmente o no, de un mismo texto, a formular hipótesis acerca de lo que podemos llamar, en términos generales, su sentido histórico y, particularmente, su función social.

Así como es importante en el trayecto interpretativo analizar, entre otros aspectos, cómo se inscriben esos instrumentos en la historia de las ideas sobre el lenguaje, qué corrientes de reflexión los orientan, con cuáles dialogan o polemizan, cuáles son las opciones teóricas y metodológicas que realizan, qué representación tienen en algunos casos del sujeto escolarizado, es central interrogar las condiciones de producción y de circulación de los textos, en lo que podemos atender a diferentes temporalidades y a marcos espaciales también distintos. Así podremos establecer lo que para nosotros tiene mayor interés: cómo en la exposición de los conocimientos sobre el lenguaje se define, con modalidades diversas, la representación que los autores tienen de la sociedad, la posición que adoptan respecto de los requerimientos de su época y cómo intervienen en la construcción de las subjetividades. 


\section{GLOTTOPOLITICAL PERSPECTIVE IN THE STUDY OF LINGUISTIC INSTRUMENTS: THEORETICAL AND ME- THODOLOGICAL ASPECTS}

\section{ABSTRACT}

This article assumes Glottopolitics as the study of the interventions in the space of language, which participate in the replication or transformation of societies. Just as these interventions depend on social processes, they also influence them from certain positions, which this analysis aims at identifying. Linguistic instruments, which regulate these practices, are considered glottopolitical gestures, and that is the reason why the conditions under which they are produced and circulate should be investigated. Within this framework and with special reference to grammars and rhetorics, this paper focuses on theoretical aspects such as the relationship between Glottopolitics and Linguistic Historiography, the temporalities which may be identified in the analysis and the spaces considered, the importance of intertext for interpretation, the social function of normative devices, and the construction of subjectivities, as well as on methodological aspects, such as the construction of series, the contrastive analysis of texts and reformulation.

KEYWORDS: Glottopolitics; linguistic instruments; grammars; rhetorics; contrastive analysis.

\section{A PERSPECTIVA GLOTOPOLITICA NO ESTUDO DOS INSTRUMENTOS LINGUÍSTICOS: ASPECTOS TEÓRICOS E METODOLÓGICOS}

\section{RESUMO}

O artigo considera a Glotopolítica como o estudo das intervenções no espaço da linguagem, que participam na reprodução ou transformação das sociedades. Assim como essas intervenções dependem dos processos sociais, incidem também neles a partir de determinadas posições que a análise procura identificar. Os instrumentos lin- 
guísticos, que se propõem regular as práticas, são enfocados como gestos glotopolíticos, daí a necessidade de atender a suas condições de produção e de circulação. Nesse marco, e com particular referência a gramáticas e retóricas, o trabalho aborda aspectos teóricos — a relação da Glotopolítica com a Historiografia Linguística, as temporalidades que podem ser identificadas na análise e os espaços considerados, a importância do intertexto na interpretação, a função social dos dispositivos normativos e a construção das subjetividades - e metodológicos — a construção de séries, a análise contrastiva de textos e a reformulação.

PALAVRAS-CHAVE: Glotopolítica; instrumentos linguísticos; gramáticas; retóricas; análise contrastiva.

\section{BIBLIOGRAFÍA}

ALTMAN, Cristina. Retrospectivas e perspectivas da historiografia da lingüística no Brasil. Revista argentina de historiografia lingüística, I, 2, 2009, p. 115-136. ARNOUX, Elvira Narvaja de. Reformulación de la Grammaire de Condillac en el Curso de Humanidades Castellanas de Jovellanos. Histoire, Épistémologie, Langage, Tomo XXIII, 1, p. 127-151, 2001a.

Disciplinar desde la lengua. La Gramática Castellana de Amado Alonso y Pedro Henríquez Ureña. In: ARNOUX, Elvira N. de; TULLIO; Angela di (orgs.). Homenaje a Ofelia Kovacci, Buenos Aires: EUDEBA, 2001b, p. 53-76.

. El análisis del discurso en el pensamiento gramatical ilustrado: Jovellanos, lector de Condillac. In: ARNOUX, Elvira N. de; LUIS, Carlos (orgs.). El pensamiento ilustrado y el lenguaje, Buenos Aires: Eudeba, 2003, p. 65-99.

. Los discursos sobre la nación y el lenguaje en la formación del Estado chileno (1842-1862). Estudio glotopolítico, Partes II y III, Buenos Aires: Santiago Arcos, 2008.

. Pensamiento gramatical y periodismo: las “notas" de dos letrados hispanoamericanos en la primera década revolucionaria en Buenos Aires. Revista Letras, n. 42, p. 189-216, 2011.

- La primera gramática escolar 'general' publicada en Buenos Aires en los años de la Independencia: la Gramática Española o Principios de la Gramática General aplicados a la Lengua Castellana de Felipe Senillosa. Histoire, Episté- 
mologie, Langage. Tomo XXXIV, fascículo 2. 43-61, 2012.

. En torno a la Nueva gramática de la lengua española (Real Academia Española y Asociación de Academias de la Lengua Española). In: ARNOUX, Elvira; NOTHSTEIN, Susana (orgs.)Temas de Glotopolítica. Buenos Aires: Biblos, 2013a, p. 245-270.

. Grammar and the state in the Southern Cone in the nineteenth century. In: José Del Valle (ed.) A Political History of Spanish. The Making of a Language. Cambridge: University Press, 2013b, p. 152-166.

. La formación retórica de la elite criolla en la etapa de construcción del Estado nacional. Estudios. Revista del Centro de Estudios Avanzados. 30 años de democracia argentina (1983-2013): Fracturas y continuidades, n. 29, enerojunio 2013 (Dossier: Discursos sociales y construcción de identidades colectivas). Universidad Nacional de Córdoba, 2013c, p. 189-215,

. Las primeras gramáticas particulares del castellano como archivos de la diversidad lingüística. In: ARNOUX, Elvira; ROCA, María del Pilar. (orgs.). Del español y del portugués: lenguas, discurso y enseñanza. João Pessoa: Universidad de Paraíba, 2013d.

. Glotopolítica: delimitación del campo y discusiones actuales con particular referencia a Sudamèrica. In: ZAJÍCOVÁ, Lenka; ZÁMEC, Radim (orgs.). Lengua y política en América Latina: Perspectivas actuales. Olomouc: Univerzita Palackého v Olomouci, 2014a. p. 19-43.

. Hacia una gramática castellana para la escuela secundaria: opciones y desplazamientos a mediados del siglo XIX. Boletín de Filología, Tomo XLIX, n. 2, 2014b, p. 19-48.

. El dispositivo normativo en la Nueva gramática básica de la lengua española. In: ARNOUX, Elvira; BEIN, Roberto (orgs.). Política lingüística y enseñanza de lenguas, Buenos Aires: Biblos, 2015a. 243-268.

. El español global y la regulación de la discursividad en línea como piezas del dispositivo glotopolítico pahispánico. In: SOUSA, Socorro Claudia Tavares de; ROCA, María del Pilar (orgs.), Políticas lingüísticas declaradas, praticadas e percebidas. João Pessoa: UFPB, 2015b.

. Teaching eloquence in a transition period: Hispanic handbooks of rhetoric in the first half of the nineteenth century. Res Rhetorica, Journal of Polish Rhetorical Society, n.1, 2015c.

La reformulación interdiscursiva en los textos gramaticales: en torno a la gramática académica de 1854 (entregado para su publicación en la Revista argentina de historiografía lingüística), 2015d. 
; José Del Valle. "Las representaciones ideológicas del lenguaje. Discurso glotopolítico y panhispanismo". Spanish in Context, número especial sobre "Ideologías lingüísticas", Amsterdã, v. 7, n.1, p. 1-24, 2010.

AUROUX, Sylvain. Histoire des idées linguistiques, t.1, Lieja: Mardaga, 1989. 1994.

La révolution technologique de la grammatisation, Lieja: Mardaga, Instrumentos lingüísticos y políticas lingüísticas: la construcción del francés, Revista argentina de historiografía lingüística, I.2., p. 137-149, 2009.

BATISTA, Ronaldo de Oliveira. Introdução à historiografía da lingüística, São Paulo: Cortez Editora, 2013.

BOURDIEU, Pierre. La codificación. In Cosas dichas, Buenos Aires: Gedisa, (1988 [1987]), p. 83-92.

BRAUDEL, Fernand. Les Temps du monde. Civilisation matérielle et capitalisme, Paris: Armand Colin, 1979.

CASTRO, Edgardo. Diccionario Foucault. Temas, conceptos y autores, Buenos Aires: Siglo XXI, 2011.

DELEUZE, Gilles. La subjetivación. Curso sobre Foucault, Buenos Aires: Cactus, 2015 [1986].

FOUCAULT, Michel. Dits et écrits, v. III, Paris: Gallimard, 1994.

FOUCAULT, Michel. Defender la sociedad, Buenos Aires: Fondo de Cultur Económica, 2014.

FUNDÉU. Escribir en Internet. Guía para los nuevos medios y las redes sociales, Barcelona: Galaxia Gutenberg, 2012.

GUIMARÃES, Eduardo. Os limites do sentido: um estudo histórico e enunciativo da linguagem, Campinas, São Paulo: Pontes, 1995.

HAN, Byung-Chul. Psicopolítica, Barcelona: Herder, 2014.

KOERNER, Konrad. La Historiografía de la Lingüística. Pasado, presente, futuro. In: DORTA, Josefa; CORRALES, Cristóbal; CORBELLA, Dolores (eds.), Historiografía de la Lingüística en el ámbito hispánico. Fundamentos epistemológicos y metodológicos, Madrid: Arco Libros, 2007, p. 15-56.

ORLANDI, Eni. O Estado, a gramática, a autoria: língua e conhecimento lingüístico. Línguas e instrumentos linguísticos. Campinas, v. 4/5, p. 19-34, 2000.

QUERÉ, Louis. Le statut duel de la langue dans l'état-nation. In: VERNES, G.; BOUTET, J. (orgs.), France, pays multilingue, t. 1, París: L'Harmattan, 1987, p. $59-78$. 
REAL ACADEMIA ESPAÑOLA. Gramática de la lengua castellana. Madrid: Imprenta Nacional, 1854.

. Epítome de la Gramática de la lengua castellana dispuesto por la Real Academia Española para la primera enseñanza elemental. Madrid: Imprenta Nacional, 1857.

. Gramática de la lengua castellana. Madrid: Imprenta Nacional, 1858.

; ASOCIACIÓN DE ACADEMIAS DE LA LENGUA ESPAÑOLA. Nueva gramática Básica de la lengua española. Buenos Aires: Espasa, 2011.

SCHLIEBEN-LANGE, Brigitte. A constituição discursiva das periodizações. Línguas e instrumentos linguísticos. Campinas, v.4/5, p.45-68, 2000.

SWIGGERS, Pierre La historiografía de la lingüística: apuntes y reflexiones. Revista argentina de historiografia lingüística, I, 1, p. 67-73, 2009.

Recebido em: 22 de dezembro de 2015

Aceito em: 07 de março de 2016 